Meta

Journal des traducteurs

Translators' Journal

\title{
La didactique de la traduction à l'heure allemande
}

\section{Ioana Balacescu et Bernd Stefanink}

Volume 50, numéro 1, mars 2005

Enseignement de la traduction dans le monde

Teaching Translation Throughout the World

URI : https://id.erudit.org/iderudit/010674ar

DOI : https://doi.org/10.7202/010674ar

Aller au sommaire du numéro

Éditeur(s)

Les Presses de l'Université de Montréal

ISSN

0026-0452 (imprimé)

1492-1421 (numérique)

Découvrir la revue

Citer cet article

Balacescu, I. \& Stefanink, B. (2005). La didactique de la traduction à l'heure allemande. Meta, 50(1), 277-293. https://doi.org/10.7202/010674ar

\section{Résumé de l'article}

C'est en Allemagne que la discussion traductologique a été la plus intense. C'est là aussi qu'elle a été centrée primordialement sur l'élaboration d'une didactique. On peut y distinguer deux grands courants : l'approche analytique et l'approche intuitive. La première entretient des liens étroits avec une linguistique qui a évolué du structuralisme au cognitivisme. La seconde se situe dans la tradition des philosophes allemands, comme Wittgenstein, Heidegger et Gadamer, dont elle se réclame dans son herméneutique.
Ce document est protégé par la loi sur le droit d'auteur. L'utilisation des services d’Érudit (y compris la reproduction) est assujettie à sa politique d'utilisation que vous pouvez consulter en ligne.

https://apropos.erudit.org/fr/usagers/politique-dutilisation/ 


\title{
La didactique de la traduction à l'heure allemande
}

\author{
IOANA BALACESCU \\ Université de Craiova, Craiova, Roumanie \\ ioanadi@hotmail.com \\ BERND STEFANINK \\ Université de Bielefeld, Bielefeld, Allemagne \\ bstefanink@hotmail.com
}

\begin{abstract}
RÉSUMÉ
C'est en Allemagne que la discussion traductologique a été la plus intense. C'est là aussi qu'elle a été centrée primordialement sur l'élaboration d'une didactique. On peut y distinguer deux grands courants: l'approche analytique et l'approche intuitive. La première entretient des liens étroits avec une linguistique qui a évolué du structuralisme au cognitivisme. La seconde se situe dans la tradition des philosophes allemands, comme Wittgenstein, Heidegger et Gadamer, dont elle se réclame dans son herméneutique.
\end{abstract}

\section{ABSTRACT}

Germany is the country where the discussion in the field of translation studies has been the most intense It is also the country where this discussion was primarily directed at the teaching of translation. Two main streams can be distinguished: the analytical approach and the intuitive approach. The first is closely linked to the evolution of linguistics from structuralism to cognitivism. The second stands in the tradition of German philosophers like Wittgenstein, Heidegger and Gadamer, from whom they derive their hermeneutics.

\section{MOTS-CLÉS/KEYWORDS}

traductologie allemande, didactique de la traduction, méthodologie en traduction, intuition et analyse en traduction, Skopostheorie

German scholars have been the most active in the field of translation studies and have produced a very large and influential body of literature on the subject. (Baker 1998: 426)

The most detailed application of Nida's theory has not occurred in England or America, but in Germany, where the science of translation (Uebersetzungswissenschaft) predominates in the teaching of translation [...]. (Gentzler 1993: 60)

Ironically, the problem with all these "sciences" of translation is that they are directed primarily at teaching translators or evaluating translations, and thus are prescriptive in nature (1993: 72)

\section{Didactique de la traduction et théories}

Une didactique de la traduction est toujours fonction d'une approche théorique, particulièrement en Allemagne, où, comme nous le verrons, elle s'est vue parrainée tantôt par les philosophes, tantôt par les linguistes. Aussi serons-nous contraints d'esquisser brièvement les bases théoriques des didactiques dont nous traiterons. 
Si l'on en croit les deux témoignages cités en exergue de cet article, il semble que c'est en Allemagne que la discussion théorique soit la plus intense. Il semble également, si l'on en croit, par exemple, le témoignage de Gentzler (1993: 60, 72), que c'est là que l'exploitation de cette discussions à des fins didactiques a été poursuivie de la façon la plus systématique (pour plus de détails voir Balacescu et Stefanink 2001 et 2002). Plus bas nous prendrons position par rapport à ce que Gentzler dit à propos du caractère "prescriptif» des théories allemandes en matière de didactique.

\section{Méconnaissance de la discussion théorique en Allemagne}

Cette prise de position s'impose parce que ces dernières sont mal comprises (voir par ex. : ci-dessous Gentzler 1993 et Robinson 1997) ou encore totalement ignorées, comme chez Venuti (1998: 27), qui cite Gideon Toury comme représentant de la tendance dite «scientifique» avec laquelle on associe pourtant d'habitude l'École allemande (voir Gentzler 1993 : 60-73; Robinson 1997: 206-211). Venuti voit Toury à l'origine d'une théorie de la traduction qui considère que «translations are facts of target cultures» (1998: 27), et, là encore, cette attribution de paternité abusive semble due à une méconnaisssance de la discussion théorique menée en Allemagne. Car si Toury a effectivement défendu ces vues - en 1995 (!) -, leurs origines remontent à la réflexion théorique menée en Allemagne au début des années 1980, pour se concrétiser de la façon la plus éclatante dans Reiß/Vermeer (1984). Lorsque Venuti parle des «limitations of linguistics-oriented approaches»(1998: 21-25), qu'il trouve «repressive in their normative principles» (1998:21), il semble ignorer les principes de base de la Skopostheorie de Reiß et Vermeer (1984), qui, loin de se laisser enfermer dans la recherche pusillanime et étriquée d'un Koller $(1979)^{1}$, propose des solutions créatives en cas de maintien du Skopos et fait du changement potentiel du Skopos une partie intégrante de sa théorie, ce qui laisse tout de même une très grande liberté à la fameuse «créativité », notion sur laquelle nous reviendrons plus tard. Le fait que dans l'article qu'il consacre aux Strategies of Translation dans la Routledge Encyclopedia of Translation Studies (Baker 1998) Robinson ne mentionne en aucune façon la Skopotheorie qui pourtant fournit un background essentiel à la discussion sur les domesticating strategies, d'une part, et les foreignizing strategies, d'autre part, qu'a entrepris l'auteur, vient corroborer l'idée d'un manque de connaissances des théories allemandes.

Cette connaissance insuffisante des théories contemporaines de la traductologie allemande se retrouve chez un certain nombre d'autres auteurs anglo-saxons, notamment chez ceux qui se réclament de principes émis par ce qu'il est convenu d'appeler la «Manipulation School». S'appuyant sur une notion de «créativité » qui n'a guère été définie, ils s'opposent à ce qu'ils appellent la «science» de la traduction pratiquée en Allemagne, qu'ils réduisent à l'approche linguistique des premiers structuralistes et qu'ils jugent, de ce fait, «réductrice» et dénuée de «créativité». Une définition de la notion de "créativité» viendrait certainement clarifier un certain nombre de choses, car les recherches de Kussmaul autant que la Skopostheorie (sans parler de notre modeste contribution) illustrent largement le fait qu'ils existe une réflexion sur la créativité chez les traductologues allemands, mais une réflexion dans le respect d'une certaine déontologie du traducteur. 


\section{Deux approches fondamentalement différentes}

Fondamentalement on peut distinguer deux types d'approches dans cette discussion traductologique allemande.

1- L'approche analytique, issue des réflexions qui ont fait suite à l'échec de la traduction automatique conçue comme un "code switching» se propose d'aboutir à une traduction «objective» en élaborant des méthodes d'analyse de plus en plus rigoureuses (et sophistiquées).

2- L'approche intuitive, en revanche, fondée sur la tradition des herméneutes allemands - notamment de Hans-Georg Gadamer (lui-même redevable à Martin Heidegger) attire l'attention sur le caractère subjectif de toute traduction et les conséquences qui en découlent.

Alors que la première, à l'heure actuelle, semble perdre pied dans ses rapports avec la réalité traduisante en proposant - sous prétexte de scientificité - des exercices de dissection du texte censés en livrer le sens profond (nous pensons tout particulièrement à Gerzymisch-Arbogast et Mudersbach 1998: 47ss. et leur méthode Aspektra) sous l'action du scalpel, la seconde se voit de plus en plus confirmée dans ses démarches heuristiques - souvent qualifiées de «mystiques» - par les recherches interdisciplinaires des cognitivistes, comme nous le montrent Risku (1998) ou, tout récemment, Stolze (2003).

\section{APPROCHES ANALYTIQUES}

Les approches analytiques sont très dépendantes de l'évolution de la linguistique, évolution que la traductologie - conçue comme une «linguistique appliquée», permettant de vérifier de manière empirique le bien - fondé des théories - a sans doute beaucoup influencée en retour. C'est là sans doute une des particularités qui caractérisent cette branche de la traductologie allemande si l'on compare, par exemple, aux affirmations de Lederer (1994: 95) : "Si intéressante qu'elle soit dans l'abstrait, celleci [la linguistique du texte] n'apporte pas grand'chose à l'étude de la traduction et moins encore à son enseignement ».

\section{I.1 Les retombées du structuralisme et de la pragmatique}

Le rapport de l'ALPAC - qui a signé l'arrêt de mort des recherches sur la traduction automatique conçue comme «code switching» - avait ouvert les yeux sur la notion de «situation». Les traductologues avaient compris qu'il fallait dépasser le mot comme unité de traduction et abandonner l'analyse sémique des structuralistes pour favoriser une didactique qui mettait en évidence la notion d' "acte de langage».

C'est ainsi que le manuel de traduction conçu par Hönig et Kußmaul Strategie der Übersetzung. Ein Lehr-und Arbeitsbuch a vu le jour ${ }^{2}$. Dans ce manuel, on attire l'attention de l'apprenant sur la «kommunikative Einbettung» d'un énoncé, c'est-àdire sur sa fonction communicative. On fait ainsi prendre conscience de la valeur illocutoire qui se cache derrière une phrase comme "Y a-t-il encore de la bière dans le frigidaire?», que l'homme, confortablement installé devant son téléviseur, adresse à sa femme, espérant qu'elle va se lever pour lui procurer l'objet de ses désirs.

Malgré leur approche communicative et leur prise en considération du récepteur de la traduction, Hönig et Kußmaul restent, en 1982 (date de la première édition) 
encore très attachés au mot comme unité de traduction. Ainsi, on peut lire que le texte est considéré comme un «faisceau de facteurs situationnels réglés par des conventions» ( Textsorte als einer konventionnell geregelten Bündelung situativer Faktoren; Hönig/Kußmaul 1982: 78) et que le mot est un ensemble de traits pertinents dont certains (voire un seul seulement) sont activés dans le contexte d'un texte (1982: 94). Aussi, pour le didacticien de la traduction, le mot est considéré comme un faisceau de traits pertinents dont on enseigne l'analyse, tout en insistant sur le fait que si le mot dans le lexique est un ensemble de traits pertinents virtuels, la tâche du traducteur sera de découvrir lesquels de ces traits virtuels sont actualisés dans le contexte du texte à traduire qu'il a devant les yeux, analyse qui sera développée dans Stefanink (1993).

L'analyse sémique, liée à la notion de «situation » et la prise en considération du background socioculturel du récepteur permettent à Hönig et Kußmaul d'introduire le concept du "degré de différenciation nécessaire» dans la traduction et de mettre entre les mains de l'apprenant des stratégies susceptibles de le guider dans la traduction de mots dits «intraduisibles», parce que culturellement marqués, les culture bound words de Whorf et Sapir.

Malgré une vue (structurale) de la sémantique aujourd'hui dépassée, cette approche didactique a porté ses fruits et le livre est un bestseller, puisqu'il en est à sa cinquième édition.

\section{I.2 Fonctionnalisme (Skopostheorie) et traduction}

Si Hönig et Kußmaul ont touché à la fonction du texte, leur objectif principal a cependant été de mettre en évidence l'importance du facteur situationnel dans la traduction, sans vraiment exploiter la notion de fonction. Cette exploitation sera réservée à Katharina Reiß et Hans Vermeer qui, en 1984, publient un ouvrage qui, lui aussi, a eu une certaine fortune, puisqu'il en est à sa deuxième édition. Il s'agit de Grundlegung einer allgemeinen Translationstheorie (Fondements d'une théorie générale de la traduction). Le terme de Skoposthéorie, créé dans cet ouvrage, a constitué une étape dans la traductologie allemande et a donné lieu a de nombreuses controverses, en partie parce qu'il a été mal compris, notamment à l'étranger, ce qui oblige à une mise au point, étant donné l'importance des applications didactiques qui en découlent et vu que cette théorie est également relativement centrale dans les discussions des théories allemandes par les traductologues à l'étranger.

Les contradictions dans l'interprétation de la Skopostheorie se situent dans le cadre plus vaste d'une méconnaissance de la traductologie allemande à l'étranger. Souvent cette traductologie est réduite à l'exploitation qu'elle fait des apports de la linguistique structurale ou chomskyienne à des fins traductologiques. Ainsi, dans Gentzler (1993), sur les 13 pages consacrées à la traductologie allemande - traitée quelque peu ironiquement de «scientifique» - onze sont dédiées à Wolfram Wilss et à l'École de Leipzig. Une demi-page seulement est consacrée à la Skopostheorie. Les apports de la psycholinguistique - tels qu'ils se reflètent, par exemple, dans les travaux de Krings (1986), Lörscher (1991), Stefanink (1991) - sont totalement ignorés, les apports de la pragmatique - tels qu'ils ont été mis à contribution par Hönig/ Kussmaul (1982) - inexistants. Quant aux théories qui y sont présentées, elles le sont de façon inadéquate. Les théories de Wilss et de l'École de Leipzig sont présentées 
comme si, enracinées dans un structuralisme rigide, qui a caractérisé leurs débuts, elles n'auraient pas, elles aussi, évolué avec l'évolution des théories linguistiques.

Quant à la Skopostheorie, les façons contradictoires dont elle a pu être reçue comme le montrent les citations suivantes de Gentzler d'une part et de Robinson d'autre part - se situent entre deux pôles fondamentalement opposés qui illustrent bien à quel point les textes allemands sont mal connus. Chez Gentzler la Skopostheorie est présentée comme un attachement pusillanime à la reproduction de la fonction du texte source:

[...] they [Reiß/Vermeer] argue that translation should be governed primarily

$[\ldots]$ by the original's Skopotheorie [...] there must also be coherence between the source text and the target text, or what she [Reiß] calls intertextual coherence [...] if the derivation is consistent with the original Skopotheorie it is called faithful, and accepted as a good translation. (Gentzler 1993: 71) (c'est nous qui mettons en relief)

Chez Robinson, on peut lire, au contraire, à propos des principes en vigueur dans la Skopostheorie:

$[\ldots]$ it is exceedingly rare for a translation to be "functionally equivalent" to its original. Functional change is the normal Skopotheorie" (Robinson: 1997: 210) (c'est nous qui mettons en relief)

Dans le premier cas, on prétend qu'aux termes de la Skopostheorie le texte cible doit se soumettre servilement au Skopos du texte source; dans le deuxième cas, on affirme, au contraire, que dans le passage du texte source au texte cible c'est le changement de Skopos qui est la norme! De telles divergences extrêmes dans la réception d'une théorie soulèvent des inquiétudes.

Que disent les textes fondateurs de la Skopostheorie?

Quand on lit:

Der Skopos ist als rezipientenabhaengige Variable beschreibbar (Reiß et Vermeer 1984: 101) (= Le Skopos peut être décrit comme une variable qui est fonction du récepteur) ${ }^{3}$,

et

Der Skopos eines Translats kann, wie mehrfach hervorgehoben vom Skopotheorie des Ausgangstexts abweichen (Reiß/Vermeer 1984: 103) (= Comme nous l'avons souligné à plusieurs reprises, le "Skopos» d'une traduction peut être différent du Skopos du texte source) (c'est nous qui mettons en relief)

on ne voit pas sur quoi se fonde l'affirmation de Gentzler, concernant l'impératif de l'identité de Skopos du texte source et du texte cible, puisque cette théorie, tout au contraire, a justement libéré le traducteur de la fixation hypnotique sur le TS, pour prendre en considération les attentes du récepteur en $\mathrm{LC}^{4}$.

Pour éviter tout malentendu, Reiß et Vermeer insistent dans leur résumé sur le fait que Bei vielen Translationen wird man Skopotheoriekonstanz (Funktionskonstanz) zwischen Ausgangs- und Zieltext als Normalfall ansetzen dürfen. (= Dans beaucoup de tradcutions on pourra considérer le maintien du Skopotheorie (le maintien de la fonction) comme le cas normal.] (Reiß et Vermeer 1984: 217) (c'est nous qui mettons en relief)

Et cette fois on se demande sur quoi Robinson se fonde pour affirmer le contraire, puisque, d'après lui, c'est le changement de Skopos (= de la fonction) qui serait le cas 
normal, le maintien étant «extrêmement rare». Si Reiß/Vermeer ont certes pu dire que

Methodologisch bleibt Skopotheoriekonstanz natürlich der Sonderfall [...] (Reiß et Vermeer 1984: 217)

il faut lire cette affirmation avec une insistance sur methodologisch. Les auteurs veulent dire que d'un point de vue méthodologique (c'est-à-dire d'intégrité dans la démarche), le traducteur doit toujours partir de l'hypothèse d'un changement de fonction, même si dans la grande majorité des cas la fonction restait identique, pour être sûr de ne pas se laisser bercer par des «évidences» simplistes. Il s'agit là d'un principe déontologique, de la fidélité à une démarche, et non pas d'une observation empirique. Il ne faut pas oublier le caractère polémique, à cette époque, d'une théorie qui devait légitimer la notion de finalité comme premier critère à respecter, dans un environnement traductologique qui avait le regard fixé sur les types d'équivalences linguistiques (voir par ex. : Koller 1979). La Skopostheorie était à cette époque le pavé dans la mare!

Mais, si un changement possible de Skopos pour le TC, doit faire partie des réflexions prioritaires du traducteur, il s'agit d'une possibilité (voir Reiß et Vermeer 1984: 103), le changement de Skopos n'est pas la règle, comme le prétend Robinson, qui ne tient pas compte du kann (peut-être).

La même contradiction dans la réception de la Skopostheorie par nos deux traductologues peut-être constatée à propos de la notion de 'cohérence intertextuelle' : Gentzler la considère comme primordiale dans la Skopostheorie (1993: 71); Robinson la considère comme inexistante dans la Skopostheorie:

The main stability lost in a text-based to action-based theories is the notion of textual equivalence, which becomes a nonissue in Skopotheorie/Handlung theories (Robinson: 1997 : 311) (c'est nous qui mettons en relief).

La vérité est entre les deux: la cohérence intertextuelle n'a pas l'importance que lui attribue Gentzler, mais elle n'est pas non plus aussi absente que le voudrait Robinson. Elle figure à la cinquième et dernière place dans la liste des critères à respecter dans le cadre de l'opération traduisante, telle qu'elle est dressée par Reiß et Vermeer (1984: 119).

Pire encore: le jugement global qui clôt le chapitre que Gentzler consacre à la traductologie allemande, loin d'être une conclusion qui découlerait de la présentation qu'il a faite, tout au long de ce chapitre, arrive inopinément, sans se baser sur aucune justification dans le corps de l'article et vient surprendre le lecteur avec une description de ce que pouvait être la traductologie des romantiques allemands au $\mathrm{XIX}^{\mathrm{e}}$ siècle:

The existing "sciences of translation" tend to be largely based upon concepts rooted in religion, German idealism, archetypes, or universal language [...] The "scientific" approaches all tend to be source-oriented in nature [...] Far from being scientific, these approaches tend to hold a transcendental, utopian conception of the translation as reproducing the original. (Gentzler 1993: 72)

On croit rêver! Comment Gentzler peut-il arriver à cette vision staëlienne de la traductologie allemande, après avoir cité des noms comme Wolfram Wilss, Katharina Reiß, Hans Vermeer, et autres qui ont marqué la traductologie allemande au cours 
des 30 dernières années? Tout au plus, ces «conceptions utopiques » de la traduction se retrouvent-elles encore au début du $\mathrm{xx}^{\mathrm{e}}$ siècle chez un Walter Benjamin (1923), mais elles n'ont rien à voir dans une description des Contemporary Translation Theories allemandes, consacrées aux 30 dernières années de ce siècle!

L'ironie qui accompagne ce regard de Gentzler sur la traductologie «scientifique» allemande

- Ironically, the problem with all these «sciences» of translation is that they are directed primarily at teaching translators or evaluating translations, and thus are prescriptive in nature (1993: 72) -

n'est pas de mise. Les recherches de Krings (1986), Kussmaul (1997), Lörscher (1992) et Stefanink 1991) sur le processus de traduction à l'aide des TAPs (Thinking Aloud Protocols) n'ont rien de prescriptif. Tout au contraire! Il s'agit d'études empiriques qui écartent expressis verbis et de façon programmatrice toute idée préconçue sur les processus de traduction et qui s'inspirent en partie des principes ethnoscientifiques (voir Stefanink 1995b). Quant à leur orientation didactique ou évaluative, elles est une des retombées de ces recherches qui mérite plutôt le respect que l'ironie. Voyons quelles sont ces retombées didactiques de la Skopostheorie!

Contrairement aux affirmations d'un Venuti (1998), cité plus haut, qui nie toute ouverture sur la créativité dans la traductologie allemande en raison de sa dépendance de la linguistique, cette traductologie allemande a au contraire poursuivi les recherches sur la créativité en traduction de façon bien plus systématique que d'autres pays, comme nous le verrons plus bas. Et la Skopostheorie a joué un rôle primordial dans cette recherche, du moins chez les traductologues qui se situent dans l'approche analytique, leur permettant ainsi de venir rejoindre les vues de ceux qui se situent dans le camp de l'approche «intuitive».

\section{Une ouverture sur une didactique de la créativité}

Dans la Skopostheorie, le donneur d'ordre peut décider le maintien ou, au contraire, le changement de la fonction du texte. Selon Katharina Reiß, on peut, par exemple, changer la fonction de la Bible dans la traduction: au lieu d'y voir un texte opérationnel qui veut convaincre à devenir chrétien, on peut le traduire pour le plaisir esthétique de la lecture et en faire un texte littéraire.

Si le donneur d'ordre ne change pas la fonction du texte, le maintien de la fonction cette dernière implique que l'effet produit sur le récepteur en langue cible sera le même que l'effet produit sur le récepteur en langue source. Pour y arriver, tous les moyens sont bons: «la fin justifie les moyens» (Reiß/Vermeer 1984: 101). Quelle ouverture toute de même sur la créativité!

Reiß/Vermeer (1984) donnent un exemple frappant, qui montre jusqu'où peuvent aller les conséquences de cette conception pour la pratique:

Supposons que dans une chronique médiévale il soit question d'une comète qui apparaît dans le ciel. Pour les gens de l'époque médiévale l'apparition d'une comète indiquait l'annonce d'une guerre. Si ce texte devait être traduit pour des gens de notre époque, il faudrait tenir compte du fait que nous ne croyons plus à la valeur symbolique de la comète annonçant une guerre. Pour nous, les comètes n'ont rien de mystérieux, leur apparition et leur disparition est prévisible sur la base de calculs adéquats. (Reiß et Vermeer 1984: 115-116) 
Selon Reiß et Vermeer, une traduction qui garderait l'exemple de la comète tel quel dans la version moderne opérerait en fait un changement de fonction:

Une information qui, à l'époque médiévale, provoquait un sentiment de peur et de panique ne suscite chez le lecteur moderne qu'un sourire amusé devant cette réaction médiévale. Une traduction qui viserait au maintien de l'effet produit devrait trouver un équivalent culturel, comme, par exemple, une déclaration de guerre d'une puissance étrangère.(Reiss et Vermeer 1984: 116)

Traduire les éléments culturels est devenu partie intégrante de l'opération traduisante et ne fait plus figure d'enjolivure plus ou moins superflue! Quelles perspectives pour l'apprenti traducteur qui arrive en général avec l'idée préconçue, sa «maxime de traduction ", qu'un mot a un sens défini dans le dictionnaire et un ou plusieurs correspondants en langue cible, parmi lesquels il doit en choisir un!

\section{I.3 La linguistique du texte et ses retombées didactiques}

\section{I.3.1 Une "typologie-des-textes-pertinente-pour-le-traducteur»}

La Skopostheorie est issue d'une linguistique du texte qui place la fonction du texte au premier plan. Cette linguistique du texte a eu d'autres retombées didactiques. En 1971, Katharina Reiß en a tiré une "typologie-des-textes-pertinente-pour-la-traduction » (übersetzungsrelevante Texttypologie; Reiß 1971: 31ss.), distinguant quatre types de texte qu'elle a ensuite réduit à trois. Se basant sur les fonctions du langage établies par le linguiste Karl Bühler (1934), elle distingue des textes où l'accent est mis primordialement sur le contenu (inhaltsbetont), des textes où c'est la forme qui prime (formbetont) et des textes à caractère appellatif (appellbetont).

Il est clair qu'un texte de nature informative se traduira autrement qu'un texte à caractère appellatif, qui veut, par exemple, convaincre d'acheter un produit. Il est clair également que dans le cas de changement de fonction d'un texte comme La Bible, qu'on pourrait traduire en tant que texte appellatif pour convaincre l'incroyant ou pour maintenir le croyant dans sa foi, sera traduit autrement si le récepteur du texte veut y trouver avant tout un texte littéraire qu'il lira pour son plaisir esthétique, auquel cas c'est la forme qui retiendra primordialement l'attention du traducteur. Pour le didacticien, ce classement fournit un critère de décision précieux pour justifier jusque dans le détail ses choix traduisants face aux problèmes d'ordre microstructural de ses apprenants (voir Stefanink 2000). Si son analyse des besoins lui révèle que ses apprenants se posent la question de savoir si une explicitation culturelle dans le TC doit se faire sous forme d'annotation, entre parenthèses ou par apposition explicative, il fera trouver la réponse en fonction du type de texte: là où dans un texte informatif une annotation s'impose, celle-ci serait malvenue dans une traduction d'Astérix (solution qui avait pourtant été suggérée naïvement par un assistant en littérature française lors d'une conférence de la traductrice allemande d'Astérix).

\section{I.3.2 Une "analyse-du-texte-pertinente-pour-le-traducteur»}

Basée sur les recherches des linguistes du texte, la didactique allemande a développé une analyse-du-texte-pertinente-pour-le-traducteur (übersetzerrelevante Textanalyse; voir par ex. Hönig 1989), connue plus généralement sous la variante «pertinente- 
pour-a traduction» (übersetzungsrelevante Textanalyse chez Reiß 1984, Bühler 1984, Hönig 1986, Nord 1988), qui se réfère principalement au questionnaire de la formule de Lasswell (Who says what in which channel to whom with what effect), enrichie de questions supplémentaires: « où? » et «quand? » pour définir le lieu et le moment de la rédaction du TS. Didactiquement, ce cadre permet une prise de conscience de l'apprenant des différents facteurs impliqués dans l'opération traduisante. Autant cette analyse exhaustive du texte source peut paraître trop coûteuse pour le professionnel qui la fera plus ou moins intuitivement et d'une façon de plus en plus automatisée, selon son degré de professionnalité, autant elle est utile pour l'apprenti traducteur, qui prend ainsi conscience des différents facteurs impliqués plus ou moins inconsciemment dans l'opération traduisante.

\section{I.3.3 Analyse du texte et fonctionnalisme}

En rajoutant les questions «pourquoi?» (warum) pour questionner sur la motivation du TS et «dans quel but?» (wozu) pour définir les intentions de l'auteur, Hönig (1986) et Nord (1988) établissent le lien entre linguistique du texte et fonctionnalisme, élaborant ainsi un système de saisie analytique du texte qui, dans la didactique allemande, a fait l'objet d'une systématisation extrême, allant jusqu'à faire des sousclassements pour le «what» de la formule Lasswell en thème et contenu, présuppositions (qu'est-ce qui n'est pas dit?) et en introduisant un «comment» (wie) qui doit amener l'apprenant à réfléchir sur la forme et le style du message.

\section{L'unité de traduction}

Ce lien entre analyse du texte, d'une part, et fonctionnalisme, d'autre part, amène à jeter un regard nouveau sur un élément capital dans la didactique de la traduction: l'unité de traduction. Introduite en 1958 par Vinay et Darbelnet dans leur Stylistique comparée, elle a donné lieu à de nombreux débats concernant sa longueur matérielle jusqu'à lui dénier toute valeur objective, la faisant dépendre de la subjectivité du traducteur. Nord (1997) se détache de la traditionnelle segmentation linéaire en unités de traduction pour introduire une vision verticale, dans laquelle l'unité de traduction est constituée par tous les éléments qui ont la même fonction dans le texte et forment ainsi une unité cohérente. Parallèlement, Stefanink (1997) parle par exemple d'une isotopie de l'ironie qui est un des éléments à traduire dans le texte cité en exemple, déclarant que «traduire, c'est traduire les isotopies! ». Aussi plaide-t-il pour une sensibilisation de l'apprenant à ces isotopies.

\section{Bilan des approches analytiques en traduction}

On a pu le constater, l'approche analytique dans la didactique allemande de la traduction est intimement liée à la linguistique, une linguistique qui n'est évidemment pas restée au stade de la linguistique structuraliste qui semble être l'objet des critiques de Venuti citées en début de cet article, mais une linguistique, qui a évolué. Elle a tellement évolué que, sur certains points, ses applications traductologiques sont venues rejoindre celles des approches intuitives. En effet, des linguistes cognitivistes comme Lakoff, Schank, Langacker, Rosch, Fillmore, etc. ont pu remettre en cause nos catégorisations analytiques de la réalité, dénonçant le Myth of Objectivism in Western Philosophy and Linguistics (Lakoff et Johnson 1980: 195 ss.) au profit des Folk Models of Categorization (Lakoff 1987) selon lesquels la saisie du sens se fait de manière associative. 


\section{APPROCHES INTUITIVES}

Dans leur tentative de fonder une didactique de la traduction sur des bases «scientifiques», Gerzymisch-Arbogast et Mudersbach (1998: 16) écartent délibérément toute préoccupation concernant l'intuition et la créativité comme étant des éléments «qui se situent au delà de toute démarche systématique». Bénéficiant de crédits de recherche en vue d'élaborer une didactique de la traduction susceptible de raccourcir la durée des études à l'université de Sarrebruck, les auteurs ont tenté d'élaborer une méthode de traduction qui se baserait sur une suite de démarches reproductibles pour chaque texte, pour arriver à une «description algorithmisable» du processus de traduction qui garantirait ainsi une certaine objectivité. Ceci les amène dans une première démarche à disséquer le texte, notant, dans une première lecture, de préférence phrase par phrase, à chaque pas, les impressions que le bout de texte qu'ils viennent de lire produit sur eux, «car ces impressions ne seront plus les mêmes lors des lectures suivantes et seront ainsi à tout jamais perdues» (Gerzymisch-Arbogast et Mudersbach 1998: 47). Inutile de dire que ceci est contraire à toute lecture normale $\mathrm{du}$ texte pour laquelle, tout de même, ce texte est initialement prévu. Dans un deuxième temps, le traducteur doit établir un tableau montrant les relations entre les différents éléments du texte pour finalement, dans une troisième démarche, représenter dans un tableau synoptique le «Wissenssystem », c'est-à-dire le système de connaissances (le world knowledge pour parler en termes cognitivistes), activé par les différents éléments textuels. Autant la première démarche (appelée "Aspektra», car elle est censée fixer les différents aspects qui frappent le lecteur au fur et à mesure de sa première lecture) nous paraît condamnable, car susceptible de développer chez l'apprenant des mécanismes d'analyse contre-productifs, et autant la deuxième (appelée «Relatra» parce qu'elle doit donner une description schématique des relations entre les éléments textuels) ne nous paraît pas apporter grand-chose à la compréhension du texte aussi spectaculaire soit-elle, autant la troisième démarche (appelée «Holontra» parce qu'elle veut saisir le texte dans sa globalité) nous paraît utile, car elle attire l'attention de l'apprenant sur le réseau des associations que le lecteur peut avoir avec chacun des ces éléments textuels. Utile, cependant, à condition qu'on mette en garde l'étudiant contre l'illusion que ce faisant, il fournirait un tableau « objectif» du savoir - du «Hintergrundwissen» (Gerzymisch-Arbogast/Mudersbach 1998: 68) - actualisé par le texte.

\section{II.1 Herméneutique et didactique de la traduction}

\section{II.1.1 Les fondements théoriques}

\section{Achtsamkeit}

Cette mise en garde constitue l'un des principes fondamentaux de l'approche herméneutique et de la didactique qui en découle. En effet, les méthodes Aspektra et Holontra mises au point par Gerzymisch-Arbogast et Mudersbach illustrent respectivement, d'une part, le cul-de-sac dans lequel peut aller s'enferrer une démarche analytique poussée à outrance et, d'autre part, le dépassement nécessaire de l'analyse du texte vers une analyse des associations qui, bien entendu, seront toujours d'ordre subjectif, malgré les efforts des auteurs de Methoden des wissenschaftlichen Übersetzens pour 
établir un tableau contrastif des associations liées aux mots du texte selon les deux langues/cultures en question sur des bases qui se voudraient objectives (pour plus de détails voir Stefanink 1998a). Aussi la première tâche d'une didactique basée sur l'herméneutique sera d'enseigner la Achtsamkeit face au texte, c'est-à-dire un éveil attentif $^{5}$, terme que nous retrouvons aussi bien chez Heidegger - qui est à l'origine de l'herméneutique de Gadamer mise en application par les traductologues herméneutes allemands - que chez les chercheurs en créativité (voir par ex. Brodbeck 1999).

Les herméneutes ont, en effet, saisi un aspect de la saisie du sens qui s'est vu confirmé par les recherches récentes des cognitivistes. Ce que l'on a appelé le «cercle vicieux» des herméneutes est le dilemme fondamental dû au fait que pour comprendre un texte il faut déjà avoir dans sa mémoire une représentation conceptuelle des éléments mentionnés dans ce texte, ceci sous forme de catégories déduites, par abstraction, de notre vécu. Lorsque, au vu des éléments textuels, ces «souvenirs » sont activés, le danger, auquel nous n'échappons pas, consiste à introduire nos associations subjectives dans la saisie du sens, qui se constitue précisément dans le processus dynamique de contact entre le texte et le vécu mémorisé du récepteur de ce texte, contact que l'herméneute Gadamer a appelé la «fusion des horizons» (Horizontverschmelzung; Gadamer 1960: 283) et que les cognitivistes ont quelque peu démystifié en parlant de processus bottom up et top down. La première démarche d'une didactique basée sur l'herméneutique sera donc une mise en garde contre l'illusion de l'objectivité.

\section{Horizontverschmelzung et autopoietischer, partiell unbewusster, intuitiver Formulierungsimpuls}

À la différence, cependant, des cognitivistes, qui parlent d'un processus de Sprachverarbeitung (Rickheit/Strohner 1993), c'est-à-dire d'un processus de saisie du sens qui demande une participation active de la part du récepteur du texte, les herméneutes y voient une «impulsion de formulation intuitive» qui saisit le récepteur/ traducteur, lorsque le processus de «Horizontverschmelzung» a fonctionnné. Là où Paepcke et Forget (1981) avaient parlé d'une «intuition foudroyante», Stolze parle d'un "processus de formulation autopoïétique, partiellement inconscient», dans lequel le message, par le biais d'une "impulsion de formulation intuitive» (Stolze 2003: 302), passe de «l'enveloppe» de la LS vers celle de la LC, ce qui, selon Stolze (2003: 211) exclut une conception de la traduction comme "processus de décision».

Le Sprachspiel comme catalyseur dans la «Horizontverschmelzung» ainsi que dans le processus intuitif de formulation qui en résulte

Là où les analystes dissèquent la phrase au scalpel, les herméneutes, conscients de l'importance du côté ludique, s'en remettent au Sprachspiel Wittgensteinien, repris par l'herméneutique de Gadamer. Ce «jeu avec le langage» est le facteur qui rend possible la Horizontverschmelzung. Pour des traductologues comme Kußmaul (2000) ou Balacescu et Stefanink (2003), cette attitude ludique face au langage est la condition sine qua non de la créativité en traduction. Elle favorise, en effet, ce que des chercheurs en créativité comme de Bono ou Guilford ont appelé respectivement "pensée latérale» ou "pensée divergente». Fritz Paepcke, traductologue et fondateur de l'École de Heidelberg, compare la recherche de la «solution heureuse» d'un problème de traduction à un jeu de football au cours duquel les joueurs se font de passes 
devant les buts adverses, jusqu'à ce que l'un d'eux, à la suite d'une passe au centre bien placée, trouve l'ouverture dans la défense adverse pour marquer le but. L'importance que Paepcke attache à l'aspect ludique que comporte pour lui l'activité traduisante se reflète jusque dans le titre de son traité de traductologie, "Ouvertures sur la traduction» (Paepcke et Forget 1981), où ouvertures - en dehors du sens contextuel dans le titre - fait allusion à la scène du jeu de football évoquée ci-dessus. Quelques années plus tard il formulera catégoriquement: "Le mode sur lequel sont fabriqués les textes et leur traduction est celui du jeu» (Paepcke 1986: 126; notre traduction).

\section{II.1.2 Les retombées didactiques}

Si l'on compare les retombées didactiques de l'approche herméneutique de Stolze (2003), qui est sans aucun doute la représentante contemporaine la plus autorisée de l'approche herméneutique en Allemagne, avec celles des traductologues que nous venons de citer comme représentants de l'approche analytique, les résultats, à première vue, semblent plutôt maigres, difficiles à concrétiser sous forme d'unités d'enseignement susceptibles de constituer la base d'une progression didactique. Pas de grand tableaux synoptiques censés représenter les réseaux sémantiques du world knowledge activé par les mots du texte, ni, non plus, de système de règles algorithmiques reproductibles, susceptibles de s'appliquer à toute traduction afin d'en garantir le statut scientifique, comme le réclament Gerzymisch-Arbogast et Mudersbach (1998), et encore moins une présentation de modèles d'équivalence sous forme de patrons, comme nous les a présentés encore très récemment Henschelmann (1999), avec lesquels on pourrait apprendre à maîtriser des "compétences partielles» Übersetzungsfertigkeiten) dans l'optique de Wilss (1992). Non, pour Stolze, le traducteur doit «saisir la pensée communiquée dans le texte pour la formuler librement dans un projet créatif, délivré des entraves de l'interférence » (Stolze 2003 : 249), une conception qui n'est pas sans rappeler un élément clé de la didactique préconisée dans l'approche interprétative de l'École de Paris: la déverbalisation (Lederer 1994 et Lavault 1985). Là encore, c'est Gadamer qui fournit les fondements philosophiques d'une approche $\mathrm{du}$ sens qui préfigure en substance ce que nous retrouverons dans le terme de «déverbalisation» créé par l'École de Paris: «Savoir lire, c'est lorsque les lettres disparaissent dans l'imperceptible et que c'est le seul sens du message qui se construit» (Gadamer 1977: 63).

Au centre de cette approche nous trouvons la personne du traducteur. Rien d'étonnant pour qui connaît Stolze, puisque déjà en 1992 celle-ci annonçait qu'une bonne didactique de la traduction devait agir sur les comportements plutôt que de se perdre dans des raffinements de l'analyse textuelle exclusivement centrés sur le texte. Mais, là encore, loin de prétendre arriver à des conclusions d'ordre épistémologique afin d'en déduire une algorithmique des démarches à suivre, comme le voudraient Lörscher (1991) ou Krings (1986; qui s'en tient toutefois aux seules prétentions d'une application didactique), Stolze s'en tient, plus modestement, à faire prendre conscience à l'apprenti traducteur des préjugés qui peuvent entraver sa compréhension du texte: «Un des éléments principaux de la compétence traduisante consiste à laisser s'exprimer la vérité du texte, et non pas de l'entraver» (Stolze 2003: 208). Le traducteur doit développer une «sensibilité linguistique» et un «sens de la langue», qualités qui n’ont rien de mystérieux, mais auxquelles il peut être «entraîné». Là, il 
faut bien le dire, l'approche intuitive vient rejoindre certains aspects de l'approche analytique, dans la mesure où Stolze (2003: 308) recommande pour cet «entraînement» une sensibilisation aux caractéristiques stylistiques du texte, aux réseaux sémantiques, aux normes textuelles, aux aspects culturels. Pour le côté théorique, elle recommande vivement des connaissances en linguistique. Pour le développement du sens de la langue, notamment lorsqu'il s'agit de la rédaction du TC, elle préconise l'entraînement à la rédaction de différents types de textes (textes créatifs, textes journalistiques, textes techniques, textes administratifs, etc).

\section{II.1.3 Les principales différences entre les deux approches}

La boucle est, en quelque sorte bouclée, pourrait-on penser : dans les deux approches, on en revient finalement à sensibiliser aux caractéristiques du texte. Il existe pourtant une différence fondamentale d'ordre épistémologique (voir Stefanink 1997, qui parle d'un «tournant épistémologique», d'une epistemologische Wende) dans la traductologie allemande. Alors que, dans l'approche analytique, l'analyse du texte se situe avant la moindre tentative de traduction, elle sert, dans l'approche intuitive, surtout à justifier les solutions créatives trouvées intuitivement. De même, la place accordée aux deux démarches, analytique et intuitive, est très différente: alors que l'approche herméneutique accorde tout de même une place à l'analyse, l'approche intuitive de Gerzymisch-Arbogast et Mudersbach (1998: 16) écarte expressis verbis l'intuition de ses considérations. On pourrait dire que, dans la démarche analytique, l'analyse est centrée sur le texte, alors que dans l'approche herméneutique la démarche à laquelle préside avant tout le principe de la Achtsamkeit est centrée sur l'éducation du traducteur. Mais la plus grande différence réside dans le fait que les herméneutes, insistent sur le caractère provisoire et inachevé de toute traduction, se basant là encore sur des affirmations heideggeriennes concernant le sens, qui n'est pas dans le texte, mais créé par le lecteur (Sinnstiftung).

\section{UNE DÉMARCHE MÉTHODOLOGIQUE : L'ANALYSE PROCÉDURALE}

\section{III.1 L'analyse procédurale et l'analyse des besoins}

Terminons par une remarque d'ordre méthodologique qui caractérise la didactique de la traduction allemande plus que d'autres: l'analyse procédurale. Utilisée par Krings (1986), Lörscher (1991) et d'autres à des fins de description du comportement traduisant de l'apprenant, elle a servi à l'analyse des besoins - qui doit présider à toute démarche pédagogique, qui veut éviter que l'information passe au-dessus des têtes auxquelles elle est destinée - dans un enseignement de la traduction centré sur l'apprenant (Stefanink 1995, 2000). En effet, «il n'y a pas de tête traduisante vierge de théorie» comme l'ont démontré Balacescu et Stefanink (2003a: 36). Les théories sont simplement sous-jacentes et déterminent les comportements de façon inconsciente. L'analyse procédurale permet de faire le ménage dans les cerveaux qui, seulement alors, sont prêts à s'ouvrir à de nouvelles théories. Alors que chez Krings et Lörscher les données sont fournies par les Thinking aloud protocols, dits TAPs, de forme monologuée, Kußmaul parle d'une forme dialoguée de ses TAPs, alors que 
Stefanink se réclame directement des principes de l'analyse conversationnelle éthnométhodolgique, tels qu'ils ont été élaborés par les sociologues américains qui se sont proposés d'étudier les idées préconçues qui circulent dans les cerveaux des nonspécialistes à propos d'un domaine scientifique: de même que l'éthnomédecine examinera les projections des naïfs sur «les hommes en blanc» et les éléments y afférant, de même le terme d' «ethnotraductologie», introduit par Stefanink (1995), désigne la science qui s'occupe des «maximes de traduction» et autres idées préconçues véhiculées par les apprentis traducteurs.

\section{III.2 L'analyse procédurale et l'étude de la créativité en traduction}

Cette analyse procédurale, centrée sur l'apprenant a également favorisé l'étude d'un aspect qui est indissociablement lié à la notion si centrale de fidélité en traduction: la créativité. C'est Kußmaul (surtout 2000, mais déjà antérieurement dans plusieurs articles) qui, le premier s'est servi de l'analyse conversationnelle pour étudier la créativité en traduction, suivi de Balacescu et Stefanink (notamment 2003a; mais les recherches sont en cours, avec le but d'une publication plus importante, résumant les résultats du projet de recherche, en 2005). La banque de données fournie par les «conversations» y est analysée sous l'angle interdisciplinaire des recherches cognitivistes: chercheurs en mémoire, psycholinguistes, chercheurs en créativité, etc. Face à l'étonnement des incrédules qui trouveront paradoxal de vouloir «enseigner» la créativité, disons tout de suite qu'il ne s'agit pas de trouver un système de règles de nature algorithmique qui devraient inévitablement conduire à la créativité, mais d'étudier l'environnement et les comportements qui se sont avérés favorables à la créativité et d'en dégager les éléments fondamentaux, comme la «fluidité de la pensée», les changements de perspective ou le déplacement de la focalisation, l'aspect ludique et la fonction du jeu, etc.; éléments que, par la suite, le didacticien s'efforcera d'introduire dans son enseignement. En dépit du refus de prendre en considération l'étude de l'intuition et de la créativité de Gerzymisch-Arbogst et Mudersbach, c'est pourtant cette étude de la créativité sur fond de cognitivisme interdisciplinaire, susceptible de légitimer les hypothèses heuristiques des herméneutes qui préfigure l'orientation que prendront les recherches traductologiques en Allemagne.

\section{Conclusion}

La traductologie allemande ne trouve donc pas ses racines dans un romantisme staëlinien comme le voudrait Gentzer (1993), mais, d'une part, dans une linguistique qui prend ses sources chez Karl Bühler (1934) et dont les traductologues ont très bien suivi l'évolution, du structuralisme au cognitivisme, d'autre part, dans une herméneutique élaborée par des philosophes comme Wittgenstein, Heidegger et Gadamer, dont les hypothèses, de nature heuristique pour le traductologue, sont en voie de se trouver confirmées par les recherches des cognitivistes. Loin d'être «limitée » (Venuti 1998: 21-25), voire repressive in their normative principles (Venuti 1998: 21), la combinaison d'approches théoriques d'ordre analytique autant qu'intuitif, associée à des méthodes d'introspection appliquées à la personne du traducteur fournit à cette traductologie les outils nécessaires à l'étude du vaste champ de recherches que constitue la créativité en traduction. 


\section{NOTES}

1. Il s'agit de la première édition de 1979, qui semble malheureusement représenter pour beaucoup la traductologie allemande par excellence, sans doute à cause du titre qui annonce une somme. On se plaît à citer son inventaire d'équivalences à plusieurs niveaux (voir par ex. : Lederer 1994: 64-65), mais Koller (1979) reste beaucoup trop marqué par le structuralisme. On ignore la version Koller $\left(1992^{4)}\right.$ fondamentalement refondue avec intégration de recherches plus récentes. Dans sa bibliographie, Lederer cite Koller 1979.

2. La liste des publications de Kußmaul au tournant des années 1980 montre bien à quel point la théorie des actes de langage a inspiré sa didactique de la traduction.. On trouve ainsi des titres comme (notre traduction): «Les verbes de modalité en anglais et les actes de langage» (1977a), «Prier quelqu'un de faire quelque chose en anglais» (1977b), In fact, actually, anyway ... : indicateurs d'actes de langage en anglais parlé informel» (1978), La théorie des actes de langage. Un reader (1980).

3. Toutes les traductions dans ce texte sont nôtres.

4. Christina Schäffer, dans son article consacré à la Skopostheorie dans Baker (1998) comprend comme nous: The shift of focus away from source text production to the more independent challenges of target-text production has brought innovation to translation theory (Baker 1998: 238).

5. Par rapport à Aufmerksamkeit qui, comme Achtsamkeit, se traduit par "attention", Achtsamkeit implique une participation active de tout le corps, tandis que Aufmerksamkeit ne concerne que l'intellect.

\section{RÉFÉRENCES}

BAKer, M. (ed.) (1998): Encyclopedia of Translation Studies, London, Routledge.

Balacescu, I. et B. Stefanink (2001): «Une traductologie au service de la didactique: l'école allemande au sein de la famille traductologique, $1^{\text {ère }}$ partie», dans Le langage et l'homme. Traductologie - Textologie 36-1, p. 89-104.

Balacescu, I. et B. Stefanink (2002): «Une traductologie au service de la didactique: l'école allemande au sein de la famille traductologique, $2^{\mathrm{e}}$ partie», Le langage et l'homme. Traductologie - Textologie 37-1, p. 155-176.

Balacescu, I. et B. Stefanink (2003) : "Les modèles explicatifs de la créativité en traduction ", dans Meta 48-4, p. 509-526

Balacescu, I. et B. Stefanink (2003a) : «Une approche théorique pour la traduction poétique», dans Thiers, G. (2003): Baratti. Commentaires et réflexions sur la traduction de la poésie, Ajaccio, Albiana, p. 24-77.

Benjamin, W. (1923) : Die Aufgabe des Übersetzers, dans StöRig, H. J. (ed.) (1963), Das Problem des Übersetzens, Darmstadt, Wissenschaftliche Buchgesellschaft, p. 182-195.

Bono, E. de (1970): Lateral Thinking. A Textbook of Creativity.London, Ward Lock Educational. Brodbeck, K.-H. (1999²): Entscheidung zur Kreativität, Darmstadt, Primus.

BüHler, H. (ed.) (1985): Translators and their Position in Society: Proceedings of the Xth World Congress of FIT, Wien, Wilhelm Braumüller.

BüHLER, K. (1934) : Sprachtheorie: Die Darstellungsfunktion der Sprache, Jena, G. Fischer.

Gadamer, H.-G. (1960): Wahrheit und Methode. Grundzüge einer philosphischen Hermeneutik. Tübingen, J.C.B. Mohr.

Gadamer, H.-G. (1977): Die Aktualität des Schönen. Kunst als Spiel, Symbol und Fest, Stuttgart, Reclam.

Gentzler, Edwin (1993): Contemporary Translation Theories, London, Routledge.

Gerzymisch-Arbogast, H. und K. Mudersbach (1998): Methoden des wissenschaftichen Uebersetzens, Tuebingen, Francke.

Henschelmann, K. (1999): Problembewusstes Übersetzen. Französisch-Deutsch. Ein Arbeitsbuch, Tübingen, Narr.

HöNIG, H. (1986) : «Übersetzen zwischen Reflex und Reflexion - ein Modell der übersetzungsrelevanten Textanalyse», dans SNell-Hornby, M. (éd.) (1986): Übersetzungswissenschaft eine Neuorientierung, Tübingen, Francke, p. 230-251. 
Hönig, H. und P. Kussmaul (1982): Strategie der Übersetzung, Tübingen, Narr.

Koller, W. (1979): Einführung in die Übersetzungswissenschaf, Wiesbaden, Quelle und Meyer.

Krings, H.P. (1986): Was in den Köpfen von Übersetzern vorgeht. Eine empirische Untersuchung zur Struktur des Übersetzungsprozesses an fortgeschrittenen Französischlernern, Tübingen, Narr.

Lakoff, G. (1987): Women, Fire and Dangerous Things. What Categories Reveal about the Mind, Chicago, University of Chicago Press.

Lakoff, G. and M. Johnson (1980): Metaphors We Live by, Chicago, The University of Chicago Press.

Lederer, M. (1994): La traduction aujourd'hui, Paris, Hachette.

Lavault, E. (1985): Fonctions de la traduction en didactique des langues, Paris, Didier Érudition.

Loerscher, W. (1991): Translation Performance, Translation Process and Translation Strategies: A Psycholinguistic Investigation, Tübingen, Narr.

Nord, C. (1988): Textanalyse und Übersetzen. Theoretische Grundlagen, Methode und didaktische Anwendung einer übersetzungsrelevanten Textanalyse, Heidelberg, Groos.

Nord, C. (1997): «Functional Units in Translation», dans Mauranen, A. and Puurtinen, T.(éds.) (1997): Translation - Acquisition - Use. AfinLa Yearbook, Jyväskylä, University Press, p. 41-50.

Paepcke, F. und P. Forget (1981): Textverstehen und Übersetzen. Ouvertures sur la Traduction. Heidelberg, Groos.

PAepcke, F. (1986): Im Übersetzen leben. Übersetzen und Textvergleich, Tübingen, Narr.

Preiser, S. (1976): Kreativitätsforschung, Darmstadt, Wissenschaftliche Buchgesellschaft.

ReIss, K. (1971): Möglichkeiten und Grenzen der Übersetzungskritik. Kategorien und Kriterien für eine sachgerechte Beurteilung von Übersetzungen, Muenchen, Max Hueber.

Reiss, K. (1984) : «Methodische Fragen der übersetzungsrelevanten Textanalyse», Lebende Sprachen 1, p. 7-10.

Reiss, K. und H. Vermeer (1984): Grundlegung einer allgemeinen Translationstheorie, Tübingen, Niemeyer.

Rickheit, G. und H. Strohner (1993): Grundlagen der kognitiven Sprachverarbeitung. Modelle, Methoden, Ergebnisse, Tübingen, Francke.

Risku, H. (1998): Translatorische Kompetenz. Kognitive Grundlagen des Übersetzens als Expertenkompetenz, Tübingen, Stauffenburg.

Robinson, D. (1997): Becoming a Translator, London, Routledge.

Stefanink, B. (1991): "Vom Nutzen und der Notwendigkeit der Theorie für den universitären Übersetzungsunterricht", dans BlaAsch, H., Chaix, P. and A. Malamah-Thomas (éds.): The Role of Translation in Foreign Language Teaching. (Triangle 10), Paris, Didier, p. 59-85.

Stefanink, B. (1993): «Traduire: De la théorie à la pratique», Le français dans le monde 254, Paris, p. 65-68.

Stefanink, B. (1995a): «Le traducteur et les mots», Le français dans le monde 275, p. 38-43.

Stefanink, B. (1995b): "L'ethnotraductologie au service d'un enseignement de la traduction centré sur l'apprenant", Le langage et l'homme 4, p. 265-293.

Stefanink, B. (1997) : "“Esprit de finesse" - "Esprit de géométrie": Das Verhältnis von "Intuition” und "übersetzerrelevanter Textanalyse” beim Übersetzen", dans KelleR, R. (éd.) : Linguistik und Literaturübersetzen, Tuebingen, Narr, p. 161-184.

Stefanink, B. (1998) : «Le rendement didactique des théories de la traduction » in Journées de la francophonie, 18-20 mars 1998, Actes du colloque, Editura Universit, Alexandru Ion Cuza, 159-175.

Stefanink, B. (1998a): «Rezension von Gerzymisch-Arbogast/Mudersbach (19098) dans FLuL 27 (1998), p. 245-248.

Stefanink, B. (2000): "Analyse conversationelle et didactique de la traduction", dans Studia Romanica Posnaniensa. Vol 25/26. Poznan: Adam Mickewicz University Press, p. 283-299. (Conférence tenue au colloque: Analyse des discours: méthodologies et implications didactiques et traductologiques. Poznan 7-10 juin 1998). 
Stolze, R. (1992): Hermeneutisches Übersetzen, Tübingen, Narr.

STOlze, R. (2003): Hermeneutik und Translation, Tübingen, Narr.

Störig, H. J. (éd.) (1963/1969): Das Problem des Uebersetzens, Darmstadt, Wissenschaftliche Buchgesellschaft.

Toury, G. (1995): Descriptive Translation Studies and Beyond, Amsterdam, Benjamins.

Vinay, J.-P. et J. Darbelnet (1958): Stylistique comparée du français et de l'anglais. Méthode de traduction, Paris, Didier.

Venuti, L. (1998): The Scandals of Translation, London, Routlege.

WiLss, W. (1992): Übersetzungsfertigkeit. Annäherungen an einen komplexen übersetzungspraktischen Begriff, Tübingen, Narr. 